\title{
Updates to the Management of Chronic Lymphocytic Leukemia
}

Presented by William G. Wierda, MD, PhD

\begin{abstract}
The decision of when to start treatment for patients with chronic lymphocytic leukemia (CLL) has not markedly changed; it is triggered by parameters of active disease, such as progressive symptoms or progressive cytopenias related to bone marrow compromise from disease. How to treat patients with CLL has become less complicated, given the growing list of options for CLL, and generally depends on the patient's age, presence of comorbidities, and risk factors (such as chromosome 17 or 11 deletion). During his presentation at the NCCN 20th Annual Conference, Dr. William Wierda focused attention on many of the new kids on the therapeutic block for CLL-the CD20 monoclonal antibodies obinutuzumab and ofatumumab, the BTK inhibitor ibrutinib, the $\mathrm{PI} 3 \mathrm{~K}$ inhibitor idelalisib, and the BCL-2 inhibitor venetoclax-and reviewed some of the clinical data supporting the use of these agents in different patient populations with CLL. (J Natl Compr Canc Netw 2015;13:662-665)
\end{abstract}

"There is a lot happening with second- and subsequent-line therapies for chronic lymphocytic leukemia [CLL], and combinations with the new small molecule inhibitors and sequencing of them are the new direction that we are taking," pronounced William G. Wierda, $\mathrm{MD}, \mathrm{PhD}$, Professor and Center Medical Director in the Department of Leukemia, Division of Cancer Medicine, at the University of Texas MD Anderson Cancer

Presented by William G. Wierda, MD, PhD, Professor and Center Medical Director in the Department of Leukemia, Division of Cancer Medicine, University of Texas MD Anderson Cancer Center, Houston, Texas.

Dr. Wierda has disclosed that he has received consultant fees or honoraria from AbbVie Inc, Celgene Corporation, Emergent BioSolutions Inc., Genentech Inc., Genzyme Corporation, Gilead Sciences, GlaxoSmithKline, Merck \& Co., Inc., Pharmacylics, and Sanofi-Aventis U.S., and grants or research support from AbbVie, Ascerta, Genentech Inc., Gilead Sciences, GlaxoSmithKline, Jannsen Pharmaceutical Products, LP, Karyopharm, and Pharmacylics

Correspondence: William G. Wierda, MD, PhD, The University of Texas MD Anderson Cancer Center, Department of Leukemia, 1515 Holcombe Blvd, Unit 0428, Houston, TX 77030.

E-mail: wwierda@mdanderson.org
Center, and a panel member of the NCCN Guidelines Panel for Non-Hodgkin's Lymphomas. Chemoimmunotherapy with FCR (fludarabine, cyclophosphamide, and rituximab) remains the first-line standard of care for young fit patients with CLL, and chlorambucil with obinutuzumab for the elderly or those with comorbidities. However, the second-line small molecule inhibitor ibrutinib may be promoted in the near future to the first-line setting, he predicted, based on data from the RESONATE-2 study.

\section{To Treat or Not to Treat}

The standard approach for patients with early-stage untreated CLL is to watch and wait. "The period of observation and monitoring could be for several years," explained Dr. Wierda. The rationale behind this strategy is that early treatment has not been shown to significantly change overall outcome, particularly survival, he said. Indications for treatment include progressive symptoms such as night sweats, fatigue, weight loss, and anemia, which have not changed over the years. "The $1996 \mathrm{NCI}$ guidelines are consistent with the 2008 IWCLL guidelines in regard to when to start treatment," revealed Dr. Wierda.

Furthermore, this initial recommendation to watch and wait holds true for patients with high-risk features, such as the presence of the $17 \mathrm{p}$ deletion. "These patients are watched more closely than those who do not have these features," stated Dr. Wierda.

\section{Frontline Standards of Care}

Considerations that guide selection among first-line treatments include patient age, presence of comorbidities, and cytogenetics. For instance, FCR remains the 
standard of care for young and fit patients with CLL. The study data supporting this recommendation predominantly come from the CLL8 and CLL10 trials. ${ }^{1,2}$ In the extended follow-up of CLL8, FCR chemoimmunotherapy produced superior outcomes over the combination of fludarabine and cyclophosphamide, with improvements shown in both progression-free survival (PFS) and overall survival." "In our MD Anderson experience with FCR, approximately 60\% of patients with a mutated immunoglobulin heavy chain variable gene (IGHV) had a PFS that extended beyond 10 years," added Dr. Wierda. "This subgroup of patients has done exceptionally well, and there is now discussion about the possibility of a portion of these patients being cured with FCR."

In CLL10, although PFS favored FCR over BR (bendamustine and rituximab) by nearly 14 months ( 55.2 vs 41.7 months), there appeared to be no difference in overall survival. ${ }^{2}$ However, the clear benefit with FCR over BR was noted in patients younger than age 65; there was more myelosuppression with FCR and less clear improvement in PFS over BR for patients older than 65 . As a result of the high toxicity (myelosuppression) and infection rates in elderly fit patients who received FCR, Dr. Wierda indicated that $\mathrm{BR}$ represents an effective alternative regimen for this elderly patient population.

As for elderly or frail patients with CLL who have comorbidities, less-intense chemotherapy regi-

\begin{tabular}{|l|c|}
\hline Agent & Development Phase \\
\hline $\begin{array}{l}\text { CD20 mAb } \\
\text { Obinutuzumab } \\
\text { Ofatumumab }\end{array}$ & $\begin{array}{c}\text { Approved } \\
\text { Approved }\end{array}$ \\
\hline $\begin{array}{c}\text { BCL-2 inhibitor } \\
\text { Venetoclax (ABT/GDC-199) }\end{array}$ & Registration phase III \\
\hline BTK inhibitors & \\
Ibrutinib & Approved \\
CC-292 & Phase Ib \\
ONO-4059 & Phase I \\
ACP-196 & Phase II \\
\hline PI3K Y/ठ inhibitors & \\
Idelalisib & Approved \\
GS-9820 & Phase I \\
IPI-145 & Pending phase III \\
AMG/ACP 319 & Phase II \\
TGR-1202 & Phase I \\
SAR245408 (XL147) & Phase I \\
\hline Syk inhibitors & \\
GS-9973 & Phase II \\
Fostamatinib & Phase I/II \\
PRT-2070 & Phase I \\
\hline
\end{tabular}

Figure 1 Newer treatments for patients with chronic lymphocytic leukemia. mens than FCR, such as chlorambucil combined with a CD20 monoclonal antibody, such as obinutuzumab or ofatumumab, have received much recent attention. ${ }^{3-5}$ One clinical trial showed that the combination of obinutuzumab and chlorambucil yielded longer PFS than rituximab and chlorambucil but showed no significant difference in overall survival in older patients with higher comorbidity scores. ${ }^{3}$ However, Dr. Wierda said there did appear to be a statistically significant difference in overall survival favoring obinutuzumab plus chlorambucil over chlorambucil alone.

In the phase III COMPLEMENT 1 study, ofatumumab plus chlorambucil produced a PFS benefit of approximately 9 months compared with chlorambucil alone in patients with untreated CLL who were not considered to be appropriate candidates for fludarabine-based treatments. ${ }^{4}$ However, there is currently no difference in overall survival. Data are also emerging that support maintenance treatment with CD20 mAb, such as in the PROLONG study, with ofatumumab maintenance therapy showed more benefit than observation after chemoimmunotherapy. ${ }^{5}$

For all patients with CLL who have del(17p), ibrutinib received approval as first-line therapy approximately a year ago. Dr. Wierda discussed this agent with much potential further in the following section.

\section{Newer Salvage Therapies}

Second-line treatments for active disease center on 2 newly approved agents: the first-in-class BTK inhibitor ibrutinib and the reversible inhibitor of PI3K idelalisib.

According to the long-term follow-up study of ibrutinib in both treatment-naïve and previously treated patients with CLL and small lymphocytic leukemia, ${ }^{6,7}$ ibrutinib monotherapy produced durable responses, even in those with $\operatorname{del}(17 \mathrm{p})$, lasting more than 3 years out from treatment initiation. "Most patients responded, upward of 90\%," revealed Dr. Wierda, with most partial responses. "The results for the treatment-naïve group were impressive, with only one patient among 31 with disease progression with current follow up," he added. Furthermore, the investigators learned that the best response to ibrutinib seems to improve over time, and so Dr. Wierda emphasized the importance of patients remaining on the drug. 
Wierda

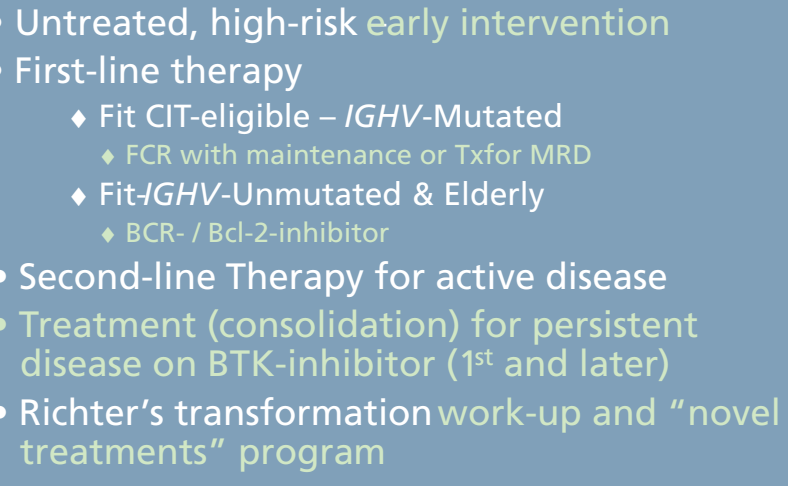

Figure 2 Chronic lymphocytic leukemia treatment directions.

Perhaps the most encouraging data have emerged from the extended follow up from the phase II and III studies, according to Dr. Wierda. ${ }^{8,9}$ In the phase II trial, ${ }^{8}$ the effectiveness of ibrutinib was demonstrated in various patient populations, including elderly patients and those with relapsed or refractory del(17p), in terms of overall response rate, duration of response, and PFS. In the phase III trial, ${ }^{9}$ where ibrutinib was compared with ofatumumab in relapsed or refractory disease, overall survival benefits with ibrutinib were shown as well.

"Results of the phase III first-line trial [RESONATE-2] comparing ibrutinib versus chlorambucil in elderly patients with CLL should be out in the next year, and we expect it to be a positive trial, showing superior results with this agent in the frontline setting," declared Dr. Wierda. Furthermore, he believes that when the data are available, its firstline indication may be expanded. "When we have the label indication and the supportive data, most patients will receive ibrutinib in the frontline setting," Dr. Wierda predicted.

Results of the second interim analysis of the phase III study data supporting the use of the combination of idelalisib and rituximab in the salvage setting were presented at the 2014 American Society of Hematology meeting. ${ }^{10}$ According to Dr. Wierda, these updated results clearly showed a statistically significant benefit with combination treatment in terms of PFS, overall response rate, and overall survival in frail patients with relapsed CLL, even in those with high-risk features. "Outcomes were similar regardless of IGHV mutation status or whether or not a patient had del(17p)," he added.

Finally, Dr. Wierda briefly reviewed results with the BCL-2 inhibitor venetoclax, which he antici- pates will be approved in the next few years. This potent monotherapy appears to be active in very highrisk relapsed patient categories (such as del[17p] and fludarabine-refractory disease). The tumor lysis events that occurred in the initial studies with this agent necessitated subsequent trial redesigns, starting with a carefully monitored step-wise escalated dose schedule. This was a better strategy for avoiding the earlier risk of rapid tumor lysis events, commented Dr. Wierda.

Early studies with venetoclax have confirmed its activity and the durability of responses in patients with high-risk CLL, including those with unmutated IGHV, del(17p), and fludarabine-refractory disease. ${ }^{11,12}$ Seymour et $\mathrm{al}^{11}$ reported response data for 78 patients, with overall survival rates of between $75 \%$ and $79 \%$. "Responses were seen similarly across the risk categories," stated Dr. Wierda. And almost one-fourth of treated patients achieved complete remission.

A smaller study of venetoclax combined with rituximab in less heavily pretreated patients with relapsed or refractory CLL was reported by Roberts et al. ${ }^{12}$ In 44 patients evaluated thus far, nearly $90 \%$ showed a response, with $34 \%$ experiencing complete remission. "For me, this study does not speak to whether or not venetoclax is better with rituximab, it speaks more to the safety and the fact that you can safely combine the agents," Dr. Wierda concluded.

\section{References}

1. 1. Fischer K, Bahlo J, Fink AM, et al. Extended follow up of the CLL8 protocol, a randomized phase III trial of the German CLL Study Group comparing fludarabine and cyclophosphamide (FC) to FC plus rituximab (FCR) for previously untreated patients with chronic lymphocytic leukemia: results on survival, progression-free survival, delayed neutropenias and secondary malignancies confirm superiority of the FCR regimen [abstract]. Blood 2012;120:Abstract 435.

2. Eichhorst B, Fink AM, Busch R, et al. Frontline chemoimmunotherapy with fludarabine, cyclophosphamide, and rituximab shows superior efficacy in comparison to bendamustine and rituximab in previously untreated and physically fit patients with advanced chronic lymphocytic leukemia: final analysis of an international, randomized study of the German CLL Study Group (CLL10 study) [abstract]. Blood 2014;124:Abstract 19.

3. Goede V, Fischer K, Busch R, et al. Obinutuzumab plus chlorambucil in patients with CLL and coexisting conditions. N Engl J Med 2014;370:1101-1110.

4. Hillmen P, Robak T, Janssens A, et al. Ofatumumab and chlorambucil versus chlorambucil alone in patients with untreated chronic lymphocytic leukemia: results of the phase III study Complement 1 [abstract]. Blood 2013;122:Abstract 528.

5. van Oers MHJ, Kuliczkowski K, Smolej L, et al. Ofatumumab maintenance prolongs PFS in relapsed CLL: prolong study interim analysis results [abstract]. Blood 2014;124:Abstract 21.

6. O'Brien SM, Furman RR, Coutre SE, et al. Independent evaluation of ibrutinib efficacy 3 years post-initiation of monotherapy in patients with 
Chronic Lymphocytic Leukemia

chronic lymphocytic leukemia/small lymphocytic leukemia including deletion 17p disease [abstract]. J Clin Oncol 2014;32:Abstract 7014.

7. Byrd JC, Furman RR, Coutre SE, et al. Three-year follow-up of treatmentnaive and previously treated patients with CLL and SLL receiving singleagent ibrutinib. Blood 2015;125:2497-1506.

8. O'Brien S, Jones JA, Coutre S, et al. Efficacy and safety of ibrutinib in patients with relapsed or refractory chronic lymphocytic leukemia or small lymphocytic leukemia with $17 \mathrm{p}$ deletion: results from the phase Il RESONATE-17 trial [abstract]. Blood 2014;124:Abstract 327.

9. Byrd JC, Brown JR, O'Brien SM, et al. Randomized comparison of ibrutinib versus ofatumumab in relapsed or refractory chronic lymphocytic leukemia/ small lymphocytic lymphoma: results from the phase III RESONATE trial [abstract]. J Clin Oncol 2014;32:Abstract LBA7008.
10. Sharman JP, Coutre SE, Furman RR, et al. Second interim analysis of a phase 3 study of idelalisib (Zydelig) plus rituximab for relapsed chronic lymphocytic leukemia: efficacy analysis in patient subpopulations with del $(17 \mathrm{p})$ and other adverse prognostic factors [abstract]. Blood 2014;124:Abstract 330

11. Seymour JF, Davids MS, Pagel JM, et al. ABT-199 (GDC-0199) in relapsed/refractory chronic lymphocytic leukemia and small lymphocytic lymphoma: high response rates among patients with high risk disease features including unmutated IGHV [abstract]. 2014 European Hematology Association Meeting. Abstract S702. Presented June 14, 2014.

12. Roberts AW, Ma S, Brander DM, et al. Determination of recommended phase 2 dose of ABT-199 (GDC-0199) combined with rituximab in patients with relapsed/refractory chronic lymphocytic leukemia [abstract]. Blood 2014;124:Abstract 325. 